\title{
Nitrogen hydrides in interstellar gas
}

\section{Herschel ${ }^{\star} / \mathrm{HIFI}$ observations towards G10.6-0.4 (W31C) ${ }^{\star \star}$}

C. M. Persson ${ }^{1}$, J. H. Black ${ }^{1}$, J. Cernicharo ${ }^{2}$, J. R. Goicoechea ${ }^{2}$, G. E. Hassel ${ }^{3}$, E. Herbst ${ }^{4}$, M. Gerin $^{5}$, M. De Luca ${ }^{5}$, T. A. Bell ${ }^{6}$, A. Coutens ${ }^{7,8}$, E. Falgarone ${ }^{5}$, P. F. Goldsmith ${ }^{9}$, H. Gupta ${ }^{9}$, M. Kaźmierczak ${ }^{10}$, D. C. Lis ${ }^{6}$, B. Mookerjea ${ }^{11}$, D. A. Neufeld ${ }^{12}$, J. Pearson ${ }^{9}$, T. G. Phillips ${ }^{6}$, P. Sonnentrucker ${ }^{12}$, J. Stutzki ${ }^{13}$, C. Vastel ${ }^{7,8}$, S. Yu $^{9}$, F. Boulanger $^{14}$, E. Dartois ${ }^{14}$, P. Encrenaz ${ }^{5}$, T. R. Geballe ${ }^{15}$, T. Giesen ${ }^{13}$, B. Godard ${ }^{5}$, C. Gry ${ }^{16}$, P. Hennebelle ${ }^{5}$, P. Hily-Blant ${ }^{17}$, C. Joblin ${ }^{7}$, R. Kołos ${ }^{18}$, J. Krełowski ${ }^{10}$, J. Martín-Pintado ${ }^{2}$, K. Menten ${ }^{20}$, R. Monje ${ }^{6}$, M. Perault ${ }^{5}$, R. Plume ${ }^{21}$, M. Salez ${ }^{5}$, S. Schlemmer ${ }^{13}$, M. Schmidt ${ }^{19}$, D. Teyssier ${ }^{22}$, I. Péron ${ }^{5,23}$, P. Cais ${ }^{24}$, P. Gaufre ${ }^{24}$, A. Cros ${ }^{7,8}$, L. Ravera ${ }^{7,8}$, P. Morris ${ }^{25}$, S. Lord ${ }^{25}$, and P. Planesas ${ }^{26}$

(Affiliations can be found after the references)

Received 31 May 2010 / Accepted 6 July 2010

\section{ABSTRACT}

The HIFI instrument on board the Herschel Space Observatory has been used to observe interstellar nitrogen hydrides along the sight-line towards G10.6-0.4 in order to improve our understanding of the interstellar chemistry of nitrogen. We report observations of absorption in NH $N=1 \leftarrow 0$, $J=2 \leftarrow 1$ and ortho- $\mathrm{NH}_{2} 1_{1,1} \leftarrow 0_{0,0}$. We also observed ortho- $\mathrm{NH}_{3} 1_{0} \leftarrow 0_{0}$, and $2_{0} \leftarrow 1_{0}$, para- $\mathrm{NH}_{3} 2_{1} \leftarrow 1_{1}$, and searched unsuccessfully for $\mathrm{NH}^{+}$. All detections show emission and absorption associated directly with the hot-core source itself as well as absorption by foreground material over a wide range of velocities. All spectra show similar, non-saturated, absorption features, which we attribute to diffuse molecular gas. Total column densities over the velocity range $11-54 \mathrm{~km} \mathrm{~s}^{-1}$ are estimated. The similar profiles suggest fairly uniform abundances relative to hydrogen, approximately $6 \times 10^{-9}, 3 \times 10^{-9}$, and $3 \times 10^{-9}$ for $\mathrm{NH}_{,} \mathrm{NH}_{2}$, and $\mathrm{NH}_{3}$, respectively. These abundances are discussed with reference to models of gas-phase and surface chemistry.

Key words. astrochemistry - ISM: abundances - ISM: molecules - molecular processes - submillimetre: ISM - line: formation

\section{Introduction}

Molecular hydrides are important in the chemistry of the interstellar medium since they often appear in the first steps in chains of reactions that lead to other more complex species. The production pathways of nitrogen-bearing molecules are still rather uncertain since key species, such as $\mathrm{NH}^{+}, \mathrm{NH}$, and $\mathrm{NH}_{2}$ have not been widely observed. Even the first identified polyatomic interstellar molecule, ammonia $\left(\mathrm{NH}_{3}\right)$, has been widely observed mainly in its para symmetry form, which leaves its formation mechanism poorly constrained in the diffuse molecular gas. Both gas-phase chemistry and grain surface reactions have been proposed as formation mechanisms, but clearly more observations are needed for a better understanding.

Both $\mathrm{NH}$ and $\mathrm{NH}_{2}$ are well known in comets (e.g. Swings et al. 1941; Meier et al. 1998; Feldman et al. 1993), and have been observed in stellar photospheres (e.g. Schmitt 1969; Farmer \& Norton 1989) via their electronic, vibration-rotation, and high rotational transitions. Interstellar NH was first detected in the interstellar medium by Meyer \& Roth (1991) by optical absorption

\footnotetext{
* Herschel is an ESA space observatory with science instruments provided by European-led Principal Investigator consortia and with important participation from NASA.

$\star \star$ Figures A.1 and A.2 (page 6) are only available in electronic form at http://www . aanda.org
}

spectroscopy. Subsequent observations by Crawford \& Williams (1997) and Weselak et al. (2009) have yielded six lines of sight where column densities of both $\mathrm{NH}$ and $\mathrm{H}_{2}$ are directly measured. The average value of the column density ratio in these diffuse and translucent sightlines is $N(\mathrm{NH}) / N\left(\mathrm{H}_{2}\right)=3 \times 10^{-9}$. Interstellar $\mathrm{NH}_{2}$ was first observed by van Dishoeck et al. (1993) in absorption towards Sgr B2 in three fine-structure components of the para- $\mathrm{NH}_{2} 1_{1,0}-1_{0,1}$ transition with partially resolved hyperfine structure at frequencies 461 to $469 \mathrm{GHz}$. Further absorption lines of both ortho and para forms of $\mathrm{NH}_{2}$ and $\mathrm{NH}$ were observed through use of the long-wavelength spectrometer aboard ISO (Cernicharo et al. 2000; Goicoechea et al. 2004; Polehampton et al. 2007). The ISO observations were unable to resolve the hyperfine structure of either molecule. Ammonia, $\mathrm{NH}_{3}$, was the first polyatomic molecule to be identified in interstellar space (Cheung et al. 1968) by means of its microwave inversion transitions. Although $\mathrm{NH}_{3}$ has been widely observed in dark clouds and star-forming regions, there have been very few measurements of the inversion lines in the diffuse interstellar gas (Nash 1990; Liszt et al. 2006).

With the unique capabilities of Herschel Space Observatory (Pilbratt et al. 2010) transitions between 157 and $625 \mu \mathrm{m}$ $(0.48-1.9 \mathrm{THz})$ are available with the Heterodyne Instrument for the Far-Infrared (HIFI; de Graauw et al. 2010) with very high sensitivity. This allows searches for spectrally resolved, 
Table 1. Observed transitions.

\begin{tabular}{lrcccc}
\hline \hline Species $^{a}$ & $\begin{array}{r}\text { Frequency } \\
(\mathrm{GHz})\end{array}$ & Band $^{b}$ & $\begin{array}{r}T_{\text {sys }}^{c} \\
(\mathrm{~K})\end{array}$ & $\begin{array}{c}t_{\text {int }}{ }^{d} \\
(\mathrm{~s})\end{array}$ & Transition \\
\hline $\mathrm{NH}^{+}$ & 1012.540 & $4 \mathrm{a}$ & 385 & 47 & ${ }^{2} \Pi_{1 / 2} J=3 / 2 \leftarrow 1 / 2$ \\
$\mathrm{NH}$ & 974.478 & $4 \mathrm{a}$ & 339 & 30 & $N=1 \leftarrow 0 J=2 \leftarrow 1$ \\
$o-\mathrm{NH}_{2}$ & 952.578 & $3 \mathrm{~b}$ & 230 & 15 & $1_{1,1}-0_{0,0}$ \\
$o-\mathrm{NH}_{3}$ & 572.498 & $1 \mathrm{~b}$ & 83 & 276 & $1_{0}-0_{0}$ \\
& 1214.859 & $5 \mathrm{a}$ & 1012 & 49 & $2_{0}-1_{0}$ \\
$p-\mathrm{NH}_{3}$ & 1215.245 & $5 \mathrm{a}$ & 1012 & 49 & $2_{1}-1_{1}$ \\
\hline
\end{tabular}

Notes. ${ }^{(a)}$ The $\mathrm{NH}$ and $\mathrm{NH}_{2}$ frequencies refer to the strongest hyperfine structure component; ${ }^{(b)}$ HIFI consists of 7 different mixer bands and two double sideband spectrometers. All transitions were observed in the upper sideband except $\mathrm{NH}^{+} ;{ }^{(c)}$ system temperature; ${ }^{(d)}$ the on-source time for each integration.

ground-state rotational transitions of $\mathrm{NH}^{+}, \mathrm{NH}, \mathrm{NH}_{2}$, and $\mathrm{NH}_{3}$ with the same instrument. Abundances of these species are key diagnostics for the nitrogen chemistry.

The most sensitive and model-independent method for measuring column densities of interstellar molecules is highresolution absorption line spectroscopy, which is being exploited by the Herschel PRISMAS key programme (PRobing InterStellar Molecules with Absorption line Studies). This paper presents the first observations and analysis of nitrogen hydrides along the sight-line towards the massive star-forming region G10.6-0.4 (W31C) as a part of the PRISMAS program. Observations of interstellar absorption towards seven additional submillimetre-wave continuum sources will be presented and analysed in later publications.

The ultra-compact HII region G10.6-0.4 in the star-forming W31 complex is an extremely luminous submillimetre and infrared continuum source. The source is located within the socalled $30 \mathrm{~km} \mathrm{~s}^{-1}$ arm at a kinematic distance of $4.8 \mathrm{kpc}$ (Fish et al. 2003). The gas associated directly with G10.6-0.4 is detected in the velocity range $V_{\mathrm{LSR}} \approx-15$ to $+5 \mathrm{~km} \mathrm{~s}^{-1}$, while the foreground gas along the line of sight is detected at $V_{\mathrm{LSR}} \approx 5$ to $55 \mathrm{~km} \mathrm{~s}^{-1}$. The focus of this paper is on the diffuse interstellar gas traced by the absorption lines.

\section{Observations}

The observations, which took place in March 2010, are summarised in Table 1 . The $2{ }_{0}-1_{0}$ and $2_{1}-1_{1} \mathrm{NH}_{3}$ transitions were observed in the same band. We used the dual beam switch mode and the wide band spectrometer (WBS) with a bandwidth of $4 \mathrm{GHz}$ and an effective spectral resolution of $1.1 \mathrm{MHz}$. The corresponding velocity resolution is about $0.3 \mathrm{~km} \mathrm{~s}^{-1}$ at the higher frequencies and $0.6 \mathrm{~km} \mathrm{~s}^{-1}$ at $572 \mathrm{GHz}$. In addition, simultaneous observations were performed using the high- resolution spectrometer (HRS) with an effective spectral resolution of $0.25 \mathrm{MHz}\left(\Delta v \sim 0.1 \mathrm{~km} \mathrm{~s}^{-1}\right)$ and a bandwidth of $230 \mathrm{MHz}$. Three observations were carried out with different frequency settings of the local oscillator (LO) corresponding to a change of approximately $15 \mathrm{~km} \mathrm{~s}^{-1}$ to determine the sideband origin of the lines. Two orthogonal polarisations were used during all observations.

The half-power beam width of the telescope is 37, 22, and $17^{\prime \prime}$ at 572, 953, and $1215 \mathrm{GHz}$, respectively. Pointing was centered at RA $=18^{\mathrm{h}} \cdot 10^{\mathrm{m}} \cdot 28^{\mathrm{s}} .70$, Dec $=-19^{\circ} .55^{\prime} \cdot 50^{\prime \prime}(J 2000)$. The reference beams were located $3^{\prime}$ on either side of the source and the calibration is described by Roelfsema (2010).

We have used the standard Herschel pipeline to Level 2 which provides fully calibrated spectra. The data were first

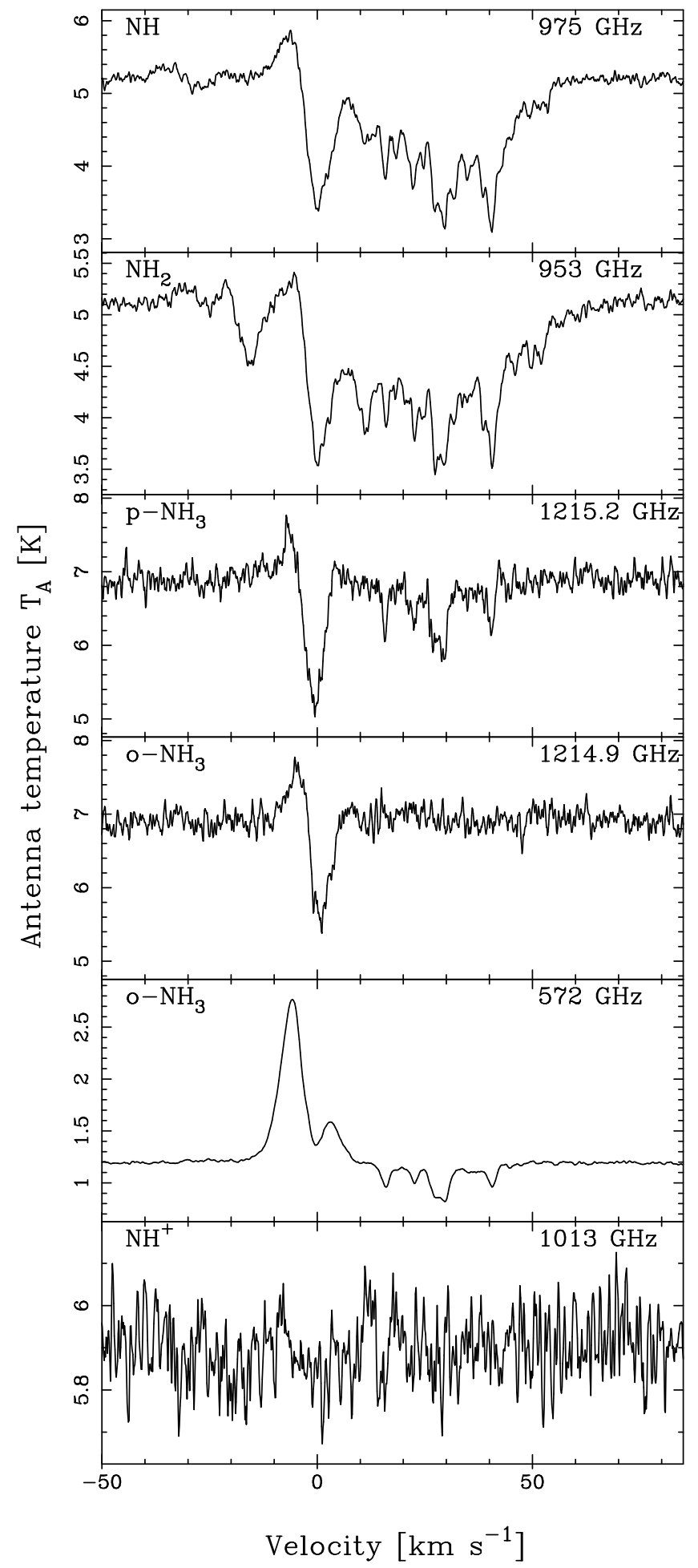

Fig. 1. Double sideband spectra of $\mathrm{NH}, o-\mathrm{NH}_{2}, o$ and $p-\mathrm{NH}_{3}$, and $\mathrm{NH}^{+}$ over the LSR velocity range -50 to $85 \mathrm{~km} \mathrm{~s}^{-1}$.

analysed using Herschel interactive processing environment (HIPE ${ }^{1}$; Ott 2010) version 2.4, and in parallel we also used the software package $\mathrm{xs}^{2}$. The data quality is excellent with very

${ }^{1}$ HIPE is a joint development by the Herschel Science Ground Segment Consortium, consisting of ESA, the NASA Herschel Science Center, and the HIFI, PACS and SPIRE consortia.

2 Developed by Per Bergman at Onsala Space Observatory, Sweden; http://www . chalmers.se/rss/oso-en/observations/ 
low intensity ripples, two polarisations that are in agreement to better than $10 \%$, and very good agreement between the three LO-tunings without contamination from the image sideband. We thus average the three LO-tunings and both polarisations in both spectra, except for $\mathrm{NH}_{3}$ at $572 \mathrm{GHz}$ and $\mathrm{NH}^{+}$which display ripples in the $\mathrm{V}$ - and $\mathrm{H}$-polarisation, respectively, and were therefore not included. The resulting rms noise is $11,120,50,51$, and $74 \mathrm{mK}$ for $\mathrm{NH}_{3}$ at 572 and $1215 \mathrm{GHz}$, for $\mathrm{NH}, \mathrm{NH}_{2}$, and $\mathrm{NH}^{+}$, respectively.

\section{Results}

Figure 1 shows the double sideband WBS spectra of all observed transitions. The frequency scale is converted as customary to Doppler velocities relative to the local standard of rest $\left(V_{\mathrm{LSR}}\right)$. All spectra, except $\mathrm{NH}^{+}$which is not detected, show emission at negative velocities and a deep absorption at $-1 \mathrm{~km} \mathrm{~s}^{-1}$ associated with G10.6-0.4 as measured by observations of $\mathrm{OH}$ masers. At higher velocities we see a number of absorption features which are also seen in atomic hydrogen (Fish et al. 2003), $\mathrm{HCO}^{+}, \mathrm{HNC}, \mathrm{HCN}$ and $\mathrm{CN}$ (Godard et al. 2010). The only transition which does not show absorption components from the foreground material is ortho- $\mathrm{NH}_{3} 2_{0}-1_{0}$.

The double-sideband calibrated antenna temperatures shown in Fig. 1 have to be divided by two in order to get the correct continuum levels, $T_{\mathrm{A}}$ (cont), which are 0.6, 2.6. 2.6, 3.0 and $3.4 \mathrm{~K}$ for $\mathrm{NH}_{3}$ at $572 \mathrm{GHz}, \mathrm{NH}_{2}, \mathrm{NH}, \mathrm{NH}^{+}$, and $\mathrm{NH}_{3}$ at $1215 \mathrm{GHz}$, respectively. The sideband gain ratio has been shown to be close to unity in previous PRISMAS observations (Neufeld et al. 2010b; Gerin et al. 2010), although departures at the $10 \%$ level have been seen at some frequencies (Neufeld et al. 2010a). We here adopt a sideband ratio of unity.

Figure 2 shows a comparison of $\mathrm{NH}, \mathrm{NH}_{2}$, and para$\mathrm{NH}_{3}$ where the intensities are normalised to the continuum in a single sideband as $T_{\mathrm{A}} / T_{\mathrm{A}}$ (cont) -1 assuming a sideband gain ratio of unity. The $\mathrm{NH}$ and $\mathrm{NH}_{2}$ spectra are strikingly similar, despite their complicated hyperfine structures. The para$\mathrm{NH}_{3}$ spectra also show the same absorption pattern, although not as strongly. Figure 3 shows a similar comparison of orthoand para- $\mathrm{NH}_{3}$. Here, too, we see the same absorption pattern in both species, and strong emission and self-absorption in the ortho-line from the source itself. The strongest velocity components in the foreground material lie at $V_{\mathrm{LSR}}=16,22.5,27.5,30$ and $40.5 \mathrm{~km} \mathrm{~s}^{-1}$.

The line opacities are calculated as $\tau=-\ln \left(T_{\text {line }} / T_{\mathrm{A}}(\mathrm{cont})-\right.$ 1). To be conservative and not include absorption from the source itself, we use the velocity interval $11-54 \mathrm{~km} \mathrm{~s}^{-1}$. The total integrated opacities are $\int \tau \mathrm{d} V=20,16,9$, and $3.4 \mathrm{~km} \mathrm{~s}^{-1}$ for $\mathrm{NH}, \mathrm{NH}_{2}, \mathrm{NH}_{3}$ at 572 and $1215 \mathrm{GHz}$, respectively.

Even though unsaturated absorption lines provide straightforward determinations of opacity and column densities, the numerous hyperfine-structure components and velocity features complicate the analysis of both $\mathrm{NH}$ and $\mathrm{NH}_{2}$. The spectroscopic properties of $\mathrm{NH}$ and $\mathrm{NH}_{2}$ have been studied extensively and references, rest frequencies and molecular properties for $\mathrm{NH}$ and $\mathrm{NH}_{2}$ are listed in the Cologne Database for Molecular Spectroscopy $^{3}$ (CDMS, Müller et al. 2005), and $\mathrm{NH}_{3}$ in Jet Propulsion Laboratory ${ }^{4}$ (JPL, Pickett et al. 1998).

In order to estimate the total column density from the integrated opacity, it is necessary to correct for the population of

\footnotetext{
3 http://www.cdms.de

4 http://spec.jpl.nasa.gov
}

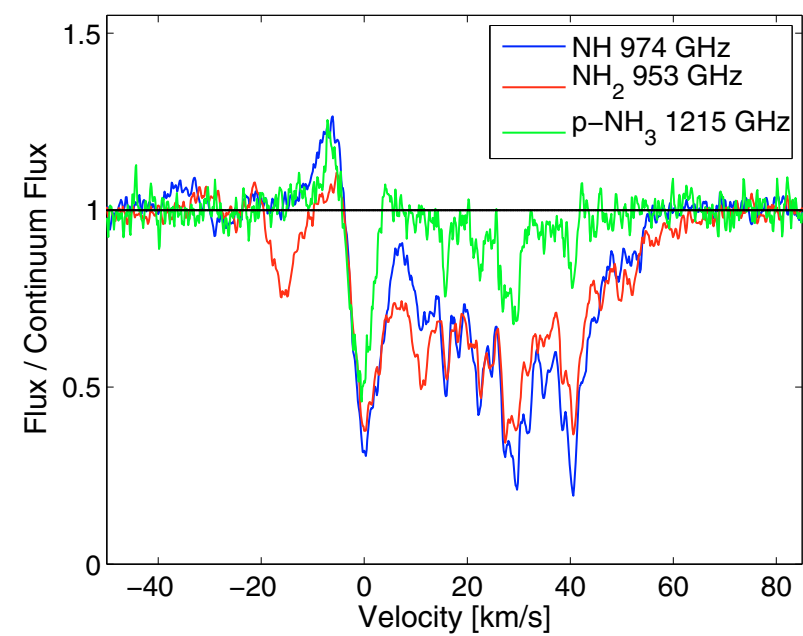

Fig. 2. Comparison of $\mathrm{NH}, o-\mathrm{NH}_{2}$ and $p-\mathrm{NH}_{3}$ at $1215.2 \mathrm{GHz}$ where the intensities have been normalised to single sideband continuum.

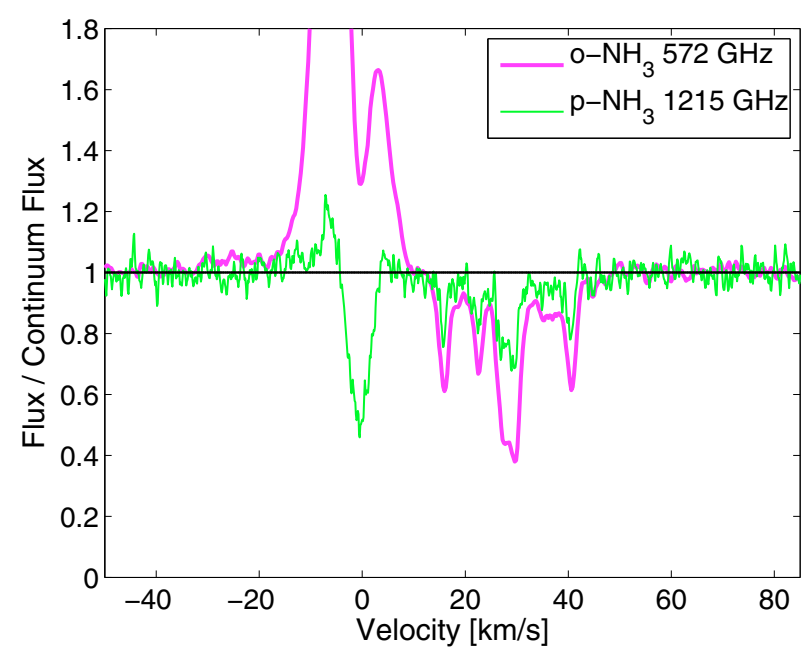

Fig. 3. Comparison of $o$ - and $p-\mathrm{NH}_{3}$ at 572.5 and $1215.2 \mathrm{GHz}$ where the intensities have been normalised to single sideband continuum.

molecules in unobserved excited levels and to quantify any possible effect of stimulated emission. We have done this through non-equilibrium excitation calculations of integrated opacity with an enhanced version of the RADEX $\operatorname{code}^{5}$ (van der Tak et al. 2007). Where collision rates are unknown, we have made guesses scaled in proportion to radiative line strengths. We assume diffuse molecular cloud conditions with $T_{\mathrm{k}}=30 \mathrm{~K}$ and $n\left(\mathrm{H}_{2}\right)=500 \mathrm{~cm}^{-3}$. The background radiation is a model of the average Galactic background radiation in the solar neighbourhood plus the cosmic microwave background radiation. The excitation of the hydride molecules in diffuse gas is dominated by the background continuum radiation and not by collisions. The resulting excitation temperatures of the observed submillimetre transitions are typically 4-5 K, which are small enough compared to $h v / k$ that no correction for emission is required. All the optical depths of the hyperfine components that contribute to the line are summed. We have treated ortho- and para-forms of molecules together in the expectation that their relative abundances are fixed by the chemical formation process. Plausible rates of formation and destruction are included explicitly in the

\footnotetext{
5 The published version will soon include these enhancements, see http://www. sron.rug.nl/ vdtak/radex/index. shtml
} 
Table 2. Total column densities, $N$, and abundances with respect to hydrogen, $X$.

\begin{tabular}{lcc}
\hline \hline Species $x$ & $\begin{array}{c}N^{a} \\
\left(\mathrm{~cm}^{-2}\right)\end{array}$ & $X=N_{x} / N_{\mathrm{H}}$ \\
\hline $\mathrm{NH}$ & $1.5 \times 10^{14}$ & $5.6 \times 10^{-9}$ \\
$\mathrm{NH}_{2}$ & $8.0 \times 10^{13}$ & $3.0 \times 10^{-9}$ \\
$\mathrm{NH}_{3}$ & $8.7 \times 10^{13}$ & $3.2 \times 10^{-9}$ \\
$\mathrm{NH}^{+}$ & $\$ 2 \times 10^{13}$ & $\lessgtr 8 \times 10^{-10}$ \\
\hline
\end{tabular}

Notes. ${ }^{(a)}$ Calculated in the velocity interval $11-54 \mathrm{~km} \mathrm{~s}^{-1}$.

excitation calculation. Thus we derive total column densities as summarised in Table 2. The analysis is complicated for $\mathrm{NH}_{3}$ by the existence of metastable excited states, especially in the para species. The entry for $\mathrm{NH}_{3}$ in Table 2 is the sum of the measured column density in $J_{K}=0_{0}$, the measured column density in the lower inversion sublevel of $1_{1}$, and calculated values in the upper inversion sublevel of $1_{1}$ and in both sublevels of $2_{2}$. All other states are expected to have negligible populations. The uncertainties of measurement and in the excitation analysis do not fully constrain the ortho/para ratio in $\mathrm{NH}_{3}$, but it appears that the effective formation temperature must be greater than $20 \mathrm{~K}$, otherwise the strength of the $1215 \mathrm{GHz}$ line cannot be explained.

We note that the three neutral nitrogen hydrides have comparable abundances: $\mathrm{NH}$ is approximately twice as abundant as $\mathrm{NH}_{2}$ and $\mathrm{NH}_{3}$. To estimate the abundance with respect to hydrogen we use $N_{\mathrm{H}}=2.7 \times 10^{22} \mathrm{~cm}^{-2}$ as total column density of hydrogen in all forms (Neufeld et al. 2010b). This value is consistent with the estimated atomic hydrogen column density in this line-of-sight, $N(\mathrm{H})=1.2 \times 10^{22} \mathrm{~cm}^{-2}$ (Godard et al. 2010) inferred from absorption profiles observed with the VLA (Fish et al. 2003), and implies that the absorbing material is mostly molecular even if there most likely are large variations between the individual velocity components. Our estimate of the abundance of NH relative to total hydrogen is somewhat higher than the $\mathrm{NH} / \mathrm{H}_{2}$ ratio of $3 \times 10^{-9}$ for six lines of sight where both are observed directly. The resulting abundances are found in Table 2 . The non-detection of $\mathrm{NH}^{+}$is used to put an upper limit on its column density and abundance $(3 \sigma)$. A previous limit on $\mathrm{NH}^{+}$ towards $\zeta$ Oph from ultraviolet observations, $N\left(\mathrm{NH}^{+}\right) / N_{\mathrm{H}}<1 \times$ $10^{-6}$, is orders of magnitude less sensitive than our limit (Snow 1979; de Almeida \& Singh 1982).

\section{Discussion}

The production of $\mathrm{NH}$ and $\mathrm{NH}_{2}$ by purely gas-phase processes is problematic since their formation is inhibited by a lack of a sufficient source of $\mathrm{N}^{+}$. The atomic ion can be formed by cosmic ray ionization of $\mathrm{N}$ or by reaction of $\mathrm{He}^{+}$with $\mathrm{N}_{2}$ or $\mathrm{CN}$, which were formed by neutral-neutral reactions. The standard gas-phase ion-molecule chemistry of nitrogen hydrides is then initiated by the reaction $\mathrm{N}^{+}+\mathrm{H}_{2} \rightarrow \mathrm{NH}^{+}+\mathrm{H}$, because $\mathrm{H}_{3}^{+}$does not react rapidly with $\mathrm{N}$. Typical steady state dark cloud models $\left(n=1 \times 10^{3}-5 \times 10^{4} \mathrm{~cm}^{-3}, T=10-40 \mathrm{~K}, A_{\mathrm{V}} \gtrsim 10\right)$, predict an $\mathrm{NH}_{2}$ abundance of $(1-10) \times 10^{-8}$, an NH abundance 10 times lower, and an $\mathrm{NH}_{2} / \mathrm{NH}_{3}$ ratio of $0.3-1.5$ for a wide range of assumptions (e.g. Millar et al. 1991; Langer \& Graedel 1989), but these are not directly applicable to diffuse molecular gas.

Previous observations of $\mathrm{NH}$ in diffuse clouds were taken to imply that NH is produced on grain surfaces (Mann \& Williams 1984; Wagenblast et al. 1993; O'Neill et al. 2002). Such models, however, often predict up to 1000 times more $\mathrm{NH}_{3}$ than $\mathrm{NH}_{2}$
(Hasegawa \& Herbst 1993). An additional source of NH and $\mathrm{NH}_{2}$ is fragmentation of ammonia by photodissociation in diffuse clouds. Models of ultraviolet illuminated photon-dominated regions predict $\mathrm{NH}_{2} / \mathrm{NH}_{3}<1$ and $\mathrm{NH}_{3} / \mathrm{NH}<1$ in regions with extinction $A_{\mathrm{v}} \lesssim 5$ mag (e.g. Sternberg \& Dalgarno 1995). The abundance patterns that we see in diffuse molecular gas are clearly different from those in Sgr B2, where $\mathrm{NH}_{3} / \mathrm{NH}_{2} / \mathrm{NH} \sim$ $100 / 10 / 1$ and the fractional abundance of $\mathrm{NH}$ is a few times $\times 10^{-9}$ (Cernicharo et al. 2000; Goicoechea et al. 2004).

We have used two different approaches to chemical models in order to compare the nitrogen-hydride abundances with theory. First, we constructed a pseudo-time-dependent model with constant physical conditions including both gas-phase and surface chemistry (Hasegawa et al. 1992) with $A_{\mathrm{V}}=2$ and $3, n_{\mathrm{H}}=$ 500 and $5000 \mathrm{~cm}^{-3}, T_{\text {gas }}=30$ and $50 \mathrm{~K}$, and $T_{\text {dust }}=10 \mathrm{~K}$. The ultraviolet field is $1 G_{0}$, and the cosmic ray ionization rate is $\zeta=$ $1.3 \times 10^{-17} \mathrm{~s}^{-1}$. The resulting abundances are found in Fig. A.1. Gas-phase chemistry alone does not match the observed abundances, failing by factors of 10 to 100 for $\mathrm{NH}$ and $\mathrm{NH}_{2}$. Inclusion of processes on dust surfaces increases the abundances of the three neutral hydrides to $10^{-8}-10^{-9}$, but fails to match the high $\mathrm{NH} / \mathrm{NH}_{3}$ ratio. If the dominant source of $\mathrm{NH}_{3}$ is association on surfaces and if the ortho/para ratio is fixed at a surface temperature below $20 \mathrm{~K}$, then the $1215 \mathrm{GHz}$ para line should be much weaker relative to the ortho $572 \mathrm{GHz}$ line than is observed. The observed line ratio is perhaps somewhat better explained by a high formation temperature, which points to exoergic gas-phase formation of $\mathrm{NH}_{3}$.

A second approach is to construct models of weak PDRs through use of the Meudon PDR code (Le Petit et al. 2006; Goicoechea \& Le Bourlot 2007), which is a steady-state, depthdependent model with pure gas-phase chemistry. We use parameters appropriate for diffuse clouds, $\mathrm{UV}=0.5-5 G_{0}, n_{\mathrm{H}}=$ $100-1000 \mathrm{~cm}^{-3}, \zeta=10^{-17}-10^{-15} \mathrm{~s}^{-1}, A_{\mathrm{V}}<5$ and illuminate one side only. The results of the best fitting model are found in Fig. A.2. Although the resulting relative abundances of nitrogenhydrides approach the observed ratios inside the cloud, the abundances relative to hydrogen are still too low.

\section{Conclusions}

Our first detections of spectrally resolved rotational transitions of nitrogen hydrides in the interstellar medium show remarkable similarities of line profiles and abundances towards one background source. The formation mechanisms are, however, difficult to explain. Neither standard gas-phase nor grain-surface chemistry can fully explain our observations, which may suggest that important reactions have been overlooked in the chemistry of interstellar nitrogen. Further analysis of the line profiles will yield abundance ratios in separate velocity components and will allow the nitrogen species to be compared directly with tracers of molecular hydrogen like $\mathrm{CH}$ and HF. Our sample will also be enlarged with seven additional sight-lines in the PRISMAS project. The chemical models will be refined and the possible role of turbulent dissipation regions will be assessed.

Acknowledgements. HIFI has been designed and built by a consortium of institutes and university departments from across Europe, Canada and the United States under the leadership of SRON Netherlands Institute for Space Research, Groningen, The Netherlands and with major contributions from Germany, France and the US. Consortium members are: Canada: CSA, U.Waterloo; France: CESR, LAB, LERMA, IRAM; Germany: KOSMA, MPIfR, MPS; Ireland, NUI Maynooth; Italy: ASI, IFSI-INAF, Osservatorio Astrofisico di Arcetri- INAF; Netherlands: SRON, TUD; Poland: CAMK, CBK; Spain: Observatorio Astronmico Nacional (IGN), Centro de Astrobiologa (CSIC-INTA). Sweden: Chalmers University of Technology - MC2, RSS \& 
GARD; Onsala Space Observatory; Swedish National Space Board, Stockholm University - Stockholm Observatory; Switzerland: ETH Zurich, FHNW; USA: Caltech, JPL, NHSC. C.P. and J.H.B. acknowledge generous support from the Swedish National Space Board. J.C. and J.R.G. thanks spanish MICINN for funding support under projects AYA2009-07304 and CSD2009-00038 M.S. acknowledges support from grant N 203393334 from Polish MNiSW.

\section{References}

Cernicharo, J., Goicoechea, J. R., \& Caux, E. 2000, ApJ, 534, L199

Cheung, A. C., Rank, D. M., Townes, C. H., Thornton, D. D., \& Welch, W. J. 1968, Phys. Rev. Lett., 21, 1701

Crawford, I. A., \& Williams, D. A. 1997, MNRAS, 291, L53

de Almeida, A. A., \& Singh, P. D. 1982, A\&A, 113, 199

de Graauw, Th., Helmich, F. P., Phillips, T. G., et al. 2010, A\&A, 518, L4

Farmer, C. B., \& Norton, R. H. 1989, A high-resolution atlas of the infrared spectrum of the sun and the earth atmosphere from space, A compilation of ATMOS spectra of the region from 650 to $4800 \mathrm{~cm}^{-1}(2.3$ to $16 \mu \mathrm{m})$, Vol. I, The sun, ed. C. B. Farmer, \& R. H. Norton

Feldman, P. D., Fournier, K. B., Grinin, V. P., \& Zvereva, A. M. 1993, ApJ, 404, 348

Fish, V. L., Reid, M. J., Wilner, D. J., \& Churchwell, E. 2003, ApJ, 587, 701

Gerin, M., de Luca, M., Black, J., et al. 2010, A\&A, 518, L110

Godard, B., Falgarone, E., Gerin, M., Hily-Blant, P., \& De Luca, M. 2010, A\&A, 520, A20

Goicoechea, J. R., \& Le Bourlot, J. 2007, A\&A, 467, 1

Goicoechea, J. R., Rodríguez-Fernández, N. J., \& Cernicharo, J. 2004, ApJ, 600, 214

Hasegawa, T. I., \& Herbst, E. 1993, MNRAS, 263, 589

Hasegawa, T. I., Herbst, E., \& Leung, C. M. 1992, ApJS, 82, 167

Langer, W. D., \& Graedel, T. E. 1989, ApJS, 69, 241

Le Petit, F., Nehmé, C., Le Bourlot, J., \& Roueff, E. 2006, ApJS, 164, 506

Liszt, H. S., Lucas, R., \& Pety, J. 2006, A\&A, 448, 253

Mann, A. P. C., \& Williams, D. A. 1984, MNRAS, 209, 33

Meier, R., Wellnitz, D., Kim, S. J., \& A'Hearn, M. F. 1998, Icarus, 136, 268

Meyer, D. M., \& Roth, K. C. 1991, ApJ, 376, L49

Millar, T. J., Bennett, A., Rawlings, J. M. C., Brown, P. D., \& Charnley, S. B. 1991, A\&AS, 87, 585

Müller, H. S. P., Schlöder, F., Stutzki, J., \& Winnewisser, G. 2005, J. Mol. Struct., 742,215

Nash, A. G. 1990, ApJS, 72, 303

Neufeld, D. A., Goicoechea, J. R., Sonnetrucker, P., et al. 2010a, A\&A, 521, L10

Neufeld, D. A., Sonnetrucker, P., Phillips, T., et al. 2010b, A\&A, 518, L108

O'Neill, P. T., Viti, S., \& Williams, D. A. 2002, A\&A, 388, 346

Ott, S. 2010, in Astronomical Data Analysis Software and Systems XIX, ed. Y. Mizumoto, K.-I. Morita, \& M. Ohishi, ASP Conf. Ser., in press

Pickett, H. M., Poynter, I. R. L., Cohen, E. A., et al. 1998, J. Quant. Spec. Radiat. Transf., 60, 883

Pilbratt, G., Riedinger, J. R., Passvogel, T., et al. 2010, A\&A, 518, L1

Polehampton, E. T., Baluteau, J., Swinyard, B. M., et al. 2007, MNRAS, 377, 1122

Roelfsema, P. R., Helmich, F. P., Teyssier, D., et al. 2010, A\&A, submitted

Schmitt, J. L. 1969, PASP, 81, 657
Snow, Jr., T. P. 1979, Ap\&SS, 66, 453

Sternberg, A., \& Dalgarno, A. 1995, ApJS, 99, 565

Swings, P., Elvey, C. T., \& Babcock, H. W. 1941, ApJ, 94, 320

van der Tak, F. F. S., Black, J. H., Schöier, F. L., Jansen, D. J., \& van Dishoeck, E. F. 2007, A\&A, 468, 627

van Dishoeck, E. F., Jansen, D. J., Schilke, P., \& Phillips, T. G. 1993, ApJ, 416, L83

Wagenblast, R., Williams, D. A., Millar, T. J., \& Nejad, L. A. M. 1993, MNRAS, 260, 420

Weselak, T., Galazutdinov, G. A., Beletsky, Y., \& Krełowski, J. 2009, MNRAS, 400,392

1 Onsala Space Observatory, Chalmers University of Technology, 43992 Onsala, Sweden e-mail: carina.persson@chalmers . se

2 Centro de Astrobiologìa, CSIC-INTA, 28850 Madrid, Spain

3 Depts. of Physics, Ohio State Univ., USA

4 Depts. of Physics, Astronomy \& Chemistry, Ohio State Univ., USA

5 LERMA, CNRS, Observatoire de Paris and ENS, France

6 California Institute of Technology, Cahill Center for Astronomy and Astrophysics 301-17, Pasadena, CA 91125, USA

7 Centre d'Étude Spatiale des Rayonnements (CESR), Université de Toulouse [UPS], 31062 Toulouse Cedex 9, France

8 CNRS/INSU, UMR 5187, 9 Avenue du Colonel Roche, 31028 Toulouse Cedex 4, France

9 JPL, California Institute of Technology, Pasadena, USA

10 Nicolaus Copernicus University, Toruń, Poland

11 Tata Institute of Fundamental Research, Homi Bhabha Road, Mumbai 400005, India

12 The Johns Hopkins University, Baltimore, MD 21218, USA

13 I. Physikalisches Institut, University of Cologne, Germany

14 Institut d'Astrophysique Spatiale (IAS), Orsay, France

15 Gemini telescope, Hilo, Hawaii, USA

16 LAM, OAMP, Université Aix-Marseille \& CNRS, Marseille, France

17 Laboratoire d'Astrophysique de Grenoble, France

18 Institute of Physical Chemistry, PAS, Warsaw, Poland

19 Nicolaus Copernicus Astronomical Center, Torun, Poland

20 MPI für Radioastronomie, Bonn, Germany

21 Dept. of Physics \& Astronomy, University of Calgary, Canada

22 European Space Astronomy Centre, ESA, Madrid, Spain

23 Institut de Radioastronomie Millimétrique, IRAM, 300 rue de la Piscine, 38406 St. Martin d'Hères, France

24 Institute Université de Bordeaux, Laboratoire d'Astrophysique de Bordeaux, 33000 Bordeaux, France; and CNRS/INSU, UMR 5804, BP 89, 33271 Floirac Cedex, France

25 Infrared Processing and Analysis Center, California Institute of Technology, MS 100-22, Pasadena, CA 91125, USA

26 Observatorio Astronácional (IGN) and Atacama Large Millimeter/Submillimeter Array, Joint ALMA Office, Santiago, Chile 


\section{Appendix A: Figures}
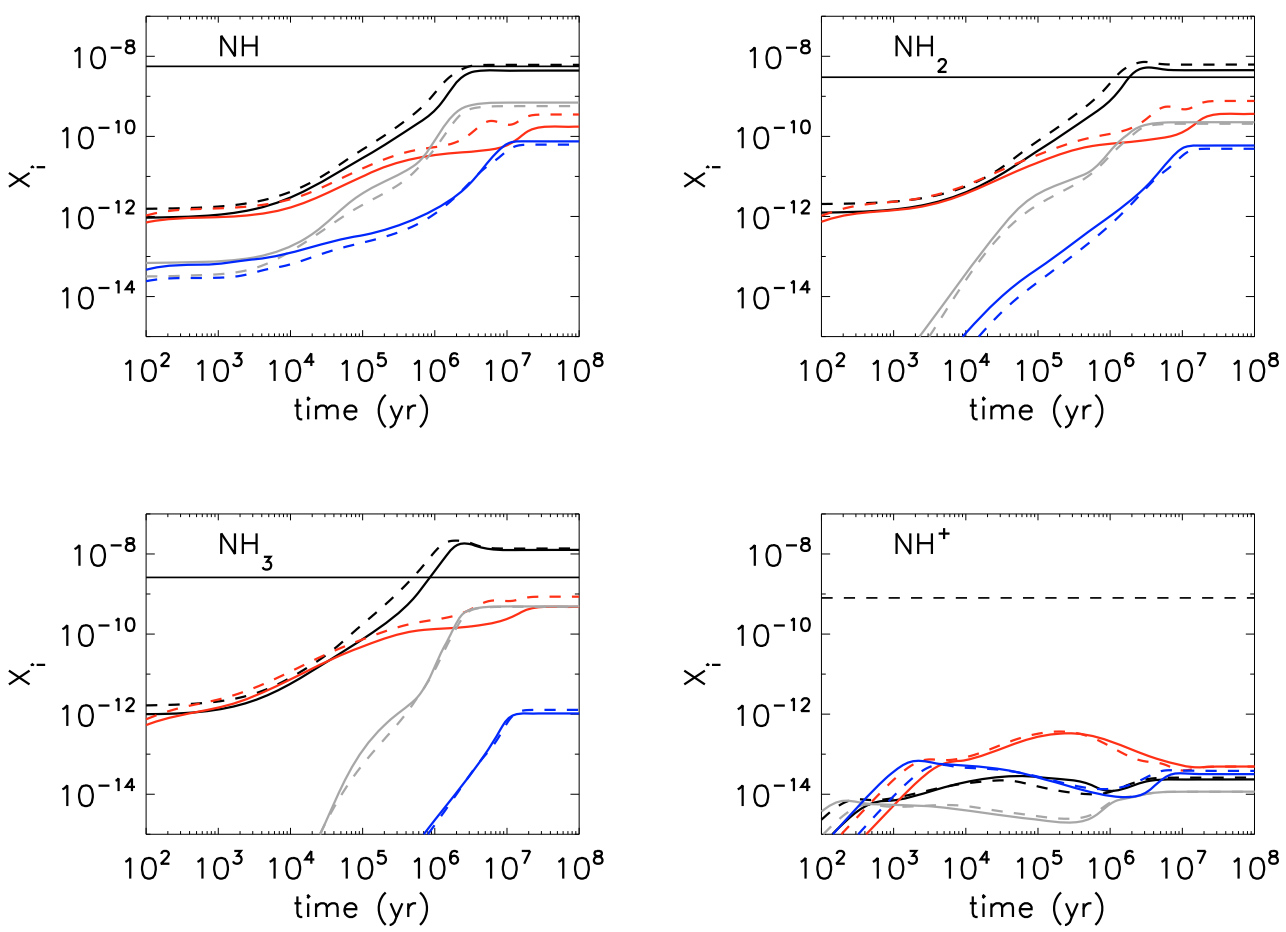

Fig. A.1. A pseudo-time-dependent model with constant physical conditions taking into account both gas-phase and grain surface chemistry. For all lines: $T_{\text {dust }}=10 \mathrm{~K}$ and the solid/dashed lines show $T_{\text {gas }}=30$ and $50 \mathrm{~K}$, respectively. The black thin lines show the observed abundances, and the dashed black line the $\mathrm{NH}^{+}$upper limit. Black line: gas phase \& grain surface chemistry using $A_{\mathrm{V}}=3, n_{\mathrm{H}}=5000 \mathrm{~cm}^{-3}$. Red line: gas phase $\&$ grain surface chemistry using $A_{\mathrm{V}}=2, n_{\mathrm{H}}=500 \mathrm{~cm}^{-3}$. Grey line: only gas phase chemistry using $A_{\mathrm{V}}=3, n_{\mathrm{H}}=5000 \mathrm{~cm}^{-3}$. Blue line: only gas phase chemistry using $A_{\mathrm{V}}=2, n_{\mathrm{H}}=500 \mathrm{~cm}^{-3}$.
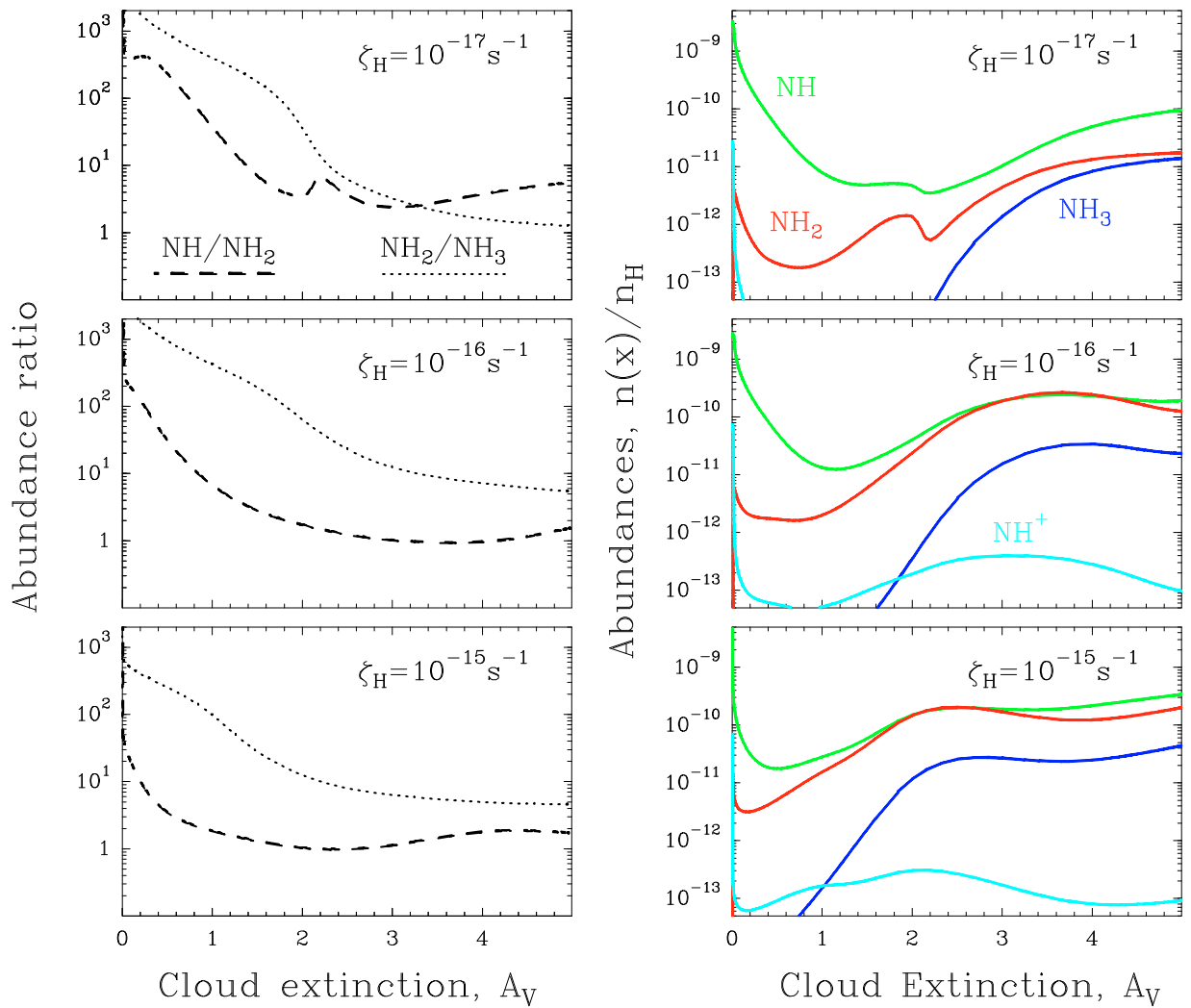

Fig. A.2. The cloud depth-dependent Meudon PDR model for pure gas-phase chemistry using $n_{\mathrm{H}}=1000 \mathrm{~cm}^{-3}, \zeta=10^{-17}-10^{-15} \mathrm{~s}^{-1}$, and a UV-field of 10 times the interstellar radiation field in Draine units $\left(\sim 5 G_{0}\right)$. The left panel shows the relative abundances of $\mathrm{NH} / \mathrm{NH}_{2}$ and $\mathrm{NH}_{2} / \mathrm{NH}_{3}$, and the right panel show the absolute abundances. 